\title{
Bases para a reforma da Atenção Primária à Saúde no Brasil em 2019: mudanças estruturantes após 25 anos do Programa de Saúde da Família
}

\author{
Bases for Brazilian Primary Health Care Reform in 2019: structural changes after 25 years of the \\ Family Health Program
}

\section{Bases para la Reforma de la Atención Primaria de Salud en Brasil en 2019: cambios estructurales después de 25 años del Programa de Salud Familiar}

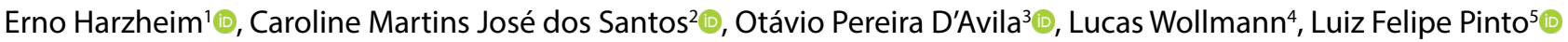 \\ ${ }^{1}$ Faculdade de Medicina, Universidade Federal do Rio Grande do Sul (UFRGS), Porto Alegre, Brasil. \\ 2 Secretaria de Atenção Primária à Saúde, Ministério da Saúde, Brasília, Brasil. \\ ${ }^{3}$ Faculdade de Odontologia, Universidade Federal de Pelotas (UFPel), Pelotas, Brasil. \\ ${ }^{4}$ Grupo Hospitalar Conceição, Porto Alegre, Brasil. \\ ${ }^{5}$ Departamento de Medicina em Atenção Primária à Saúde, Faculdade de Medicina, Universidade Federal do Rio Janeiro (UFRJ), Rio de Janeiro, Brasil
}

\section{Resumo}

O ano de 2019 marcou a estruturação das bases para uma profunda reforma na Atenção Primária à Saúde (APS) do Brasil. Os desafios enfrentados através dessa reforma foram a falta de priorização política real da APS, o financiamento insuficiente da APS e focado na estrutura de serviços, os obstáculos ao acesso de primeiro contato, a escassez de profissionais qualificados, a necessidade de maior informatização da APS e a ausência de integração de dados clínicos, a fragilidade clínica e necessidade de ampliação do escopo profissional e a falta de informação de qualidade para tomada de decisão clínica e gerencial. Com ações direcionadas a cada um desses desafios, a Secretaria de Atenção Primária à Saúde do Ministério da Saúde criou estratégias sistêmicas e integradas entre si que representam mudanças estruturantes e investimentos em governança clínica para transformar a APS brasileira, garantindo mais e melhor saúde para a população, com mecanismos transparentes e técnicos para seu financiamento, organização, oferta, monitoramento e avaliação.

Palavras-chave: Atenção Primaria à Saúde; Estratégia de Saúde da família; Ministério da Saúde; Brasil.

\begin{abstract}
The year was 2019. It marked the foundations for a profound Primary Health Care (PHC) Reform in Brazil. The challenges faced through this were the lack of real $\mathrm{PHC}$ political prioritization, insufficient $\mathrm{PHC}$ funding and focused on the service structure, obstacles to first contact access, the shortage of qualified professionals, the need for greater PHC computerization and the lack of integration of clinical data. Also, the clinical fragility and the need to expand the professional scope and the lack of quality information for clinical and managerial decision making. Actions were directed to each of these challenges, and the Ministry of Health's Secretariat of Primary Health Care created systemic and integrated strategies which represented structural changes and investments in clinical governance to transform Brazilian PHC, ensuring more and better health for the population, with transparent and technical mechanisms for its financing, organization, provision, monitoring and evaluation.
\end{abstract}

Keywords: Primary Health Care; Family Health Strategy; Ministry of Health; Brazil.

Como citar: Harzheim E, Santos CMJ, D’Avila OP, Wollmann L, Pinto LF. Bases para a reforma da Atenção Primária à Saúde no Brasil em 2019: mudanças estruturantes após 25 anos do Programa de Saúde da Família. Rev Bras Med Fam Comunidade. 2020;15(42):2354. https://doi.org/10.5712/rbmfc15(2)2354

\author{
Autor correspondente: \\ Erno Harzheim. \\ E-mail: eharzheim@hcpa.edu.br \\ Fonte de financiamento: \\ declaram não haver. \\ Parecer CEP: \\ aprovado. \\ Procedência e revisão por pares: \\ revisado por pares. \\ Recebido em: 20/01/2020. \\ Aprovado em: 24/03/2020.
}




\section{Resumen}

El año era 2019. Marcó la estructuración de las bases para una reforma profunda en la Atención Primaria de Salud (APS) en Brasil. Los desafíos enfrentados a través de esta reforma fueron la falta de una priorización política real de APS, fondos insuficientes de APS y centrados en la estructura del servicio, obstáculos para el acceso de primer contacto, la escasez de profesionales calificados, la necesidad de una mayor informatización de APS y la falta de integración de datos clínicos, la fragilidad clínica y la necesidad de ampliar el alcance profesional y la falta de información de calidad para la toma de decisiones clínicas y gerenciales. Con acciones dirigidas a cada uno de estos desafíos, la Secretaría de Atención Primaria de Salud del Ministerio de Salud creó estrategias sistémicas e integradas que representan cambios estructurales e inversiones en la gobernanza clínica para transformar la APS brasileña, garantizando más y mejor salud para la población, con mecanismos transparentes y técnicos para su financiación, organización, provisión, seguimiento y evaluación.

Palabras clave: Atención Primaria de Salud; Estrategia de Salud Familiar; Ministerio de Salud; Brasil.

\section{INTRODUÇÃO}

Neste artigo são apresentadas e analisadas as principais propostas governamentais em curso desde 2019 para a transformação das políticas públicas em atenção primária à saúde (APS) no Brasil, naquilo que intitulamos as bases estruturantes para uma Reforma na APS do Brasil. O objetivo principal da reforma é fortalecer a APS brasileira na direção da garantia dos atributos essenciais e derivados da APS segundo evidências robustas consolidadas por Starfield et al. ${ }^{1}$ Esta reforma foi necessária a fim de iniciar o processo de correção das inúmeras disfunções que o Sistema Único de Saúde (SUS) apresenta, após 25 anos da criação do então Programa de Saúde da Família em 1994. Essas disfunções fazem com que o sistema não consiga atender às necessidades e expectativas da população na mesma ordem do montante de recursos públicos que consome.

A discussão permanente sobre o subfinanciamento do SUS não faz parte desta análise, nem das definições políticas tomadas, visto que vivemos na maior crise econômica da história da República Brasileira e aumentar o emprego de recursos públicos, neste momento, seria um erro frente à sociedade atual, ${ }^{2}$ um equívoco ainda mais grave frente à herança que deixaremos às gerações futuras. A reforma foi fundamentada na opção estratégica da melhor gestão possível dentro de um orçamento global do SUS estável, com crescimento anual igual à variação do Índice Nacional de Preços ao Consumidor Amplo (IPCA) - que no ano de 2018 foi de $3,75 \%$ e no ano de 2019 foi de $4,31 \%$ - em que era fundamental ampliar os recursos para a APS.

Dois conjuntos de princípios orientaram as decisões políticas e de gestão que foram tomadas pelo Ministério da Saúde e pela Secretaria de Atenção Primária à Saúde (SAPS), criada em 17 de maio de 2019. ${ }^{3}$ O primeiro deles se relaciona a uma visão contemporânea, democrática e liberal da necessidade de reforma do Estado brasileiro. Os princípios orientadores desta visão são o livre-arbítrio, a subsidiariedade, a autonomia da gestão municipal, uma menor regulação e planificação central, a diminuição de barreiras normativas, a valorização da criatividade e do empreendedorismo, a ênfase no aumento da produção de serviços e o foco total nos resultados e na qualidade do serviço prestado. Cada real coletado de impostos tem que se traduzir em serviços de qualidade para o cidadão ou, caso contrário, este imposto deve deixar de existir. Portanto, é dever irrevogável de cada agente público do Brasil honrar os tributos que, em nosso país em particular, têm grande peso sobre a renda familiar de cada indivíduo a ser coberto pelos serviços do SUS. 
Esses princípios podem muito bem ser aplicados ao sistema público universal de saúde, principalmente ao nos mantermos firmes ao nosso texto constitucional que afirma que a saúde é um dever do Estado brasileiro.

O segundo conjunto de princípios diz respeito às características de um sistema universal de saúde efetivo, equânime e orientado pela APS. Esses princípios são a centralização do sistema nas pessoas, a ordenação do sistema por uma APS forte com presença intensa de seus clássicos atributos essenciais e derivados e a valorização da clínica dos profissionais de saúde. Além disso, seu modelo de financiamento, monitoramento e avaliação deve induzir o alcance de resultados esperados pela sociedade; as decisões devem ter forte embasamento científico; promover o uso intensivo da melhor tecnologia efetiva disponível; e focar na busca dos melhores resultados em saúde, com qualidade, transparência e equidade.

Após a definição desses dois conjuntos de princípios, antes de planejar a reforma era necessário ter um diagnóstico situacional robusto sobre as conquistas, os fracassos e as disfunções do SUS e, particularmente, da APS no SUS.

As conquistas do SUS são inúmeras. Em 30 anos criou-se um grande emaranhado de serviços de saúde, da APS aos hospitais ditos de $4^{a}$ geração, passando por serviços ambulatoriais especializados, centros oncológicos, oferta de transplantes, ações intersetoriais de promoção de saúde e de enfrentamento dos determinantes mais distais do adoecimento e de promoção dos determinantes de maior qualidade de vida. Essa oferta se traduziu na diminuição da mortalidade infantil e materna, no aumento da longevidade, na expansão da rede de serviços, na mudança do perfil epidemiológico, entre outras. Obviamente, esses avanços não são exclusivamente dependentes do SUS. São, também, consequências da evolução da sociedade brasileira nos últimos 30 anos, com redução dos níveis absolutos da pobreza, aumento dos índices de alfabetização, entre outras políticas sociais, com as de renda mínima, aposentadoria rural, etc.

Em relação aos fracassos do SUS temos dois grupos principais. O primeiro é o do acesso. Por mais que a rede de serviços tenha se ampliado - 43.275 equipes de Saúde da Família, ao redor de 6.000 hospitais gerais e especializados e cerca de 300 mil leitos hospitalares em dezembro/2019, uma das maiores características negativas do SUS é a restrição ao acesso. Temos dificuldade de acesso inclusive à APS. O atributo acesso de primeiro contato é sempre o pior avaliado nas inúmeras pesquisas já realizadas. ${ }^{4,5,6}$ Temos também listas de espera para consultas especializadas, para exames de imagem e laboratoriais, para internações e para procedimentos cirúrgicos de baixa complexidade.

O segundo grupo de fracassos é a insuficiente qualidade no manejo dos fatores de risco e das condições crônicas, incluindo as de saúde mental. Nos últimos 10 anos, apesar do avanço diagnóstico e terapêutico, seguimos com baixas taxas de controle das principais doenças crônicas (hipertensão arterial, diabetes, cardiopatia isquêmica, asma, doença pulmonar crônica obstrutiva (DPOC), problemas osteomusculares) ${ }^{7,8}$ e vimos o aumento dos problemas de saúde mental causarem inúmeros prejuízos sociais, principalmente para os indivíduos e suas famílias, mas também para o universo produtivo do emprego e da renda. Paralela e contraditoriamente, por exemplo, houve aumento considerável da oferta da estrutura dos serviços habilitados (1.485 para 3.062 CAPS entre dezembro/2008 e dezembro/2019) sem efeito na carga das doenças mentais, com taxas inadmissíveis de reinternações e tendo o consumo prejudicial do álcool como o primeiro fator de risco modificável, como apontado pelo Global Burden of Diseases. ${ }^{9}$ 
Esse conjunto de sucessos e fracassos apontam de maneira objetiva para a disfuncionalidade do SUS: há um número expressivo de hospitais de grande porte com incorporação tecnológica de alta densidade e boa qualidade assistencial, sem condições nem adequação para absorver o aumento da demanda assistencial. Concomitantemente, temos serviços especializados ambulatoriais insuficientes com qualidade heterogênea, resolutividade limitada, baixa incorporação tecnológica e cujo acesso é praticamente desvinculado de mecanismos de regulação clínica e de coordenação assistencial, aliados a inúmeras barreiras de acesso. E, onde deveria estar a condução do sistema, temos serviços de APS com qualidade heterogênea, resolutividade limitada, baixa incorporação tecnológica e flagrante ausência de mecanismos de coordenação assistencial aliados a dificuldades de acesso. Como se não bastasse, temos mecanismos de financiamento, principalmente dos serviços especializados, baseados na lógica do pagamento por procedimento realizado (chamado de fee for service) e de incentivos financeiros centralmente definidos e alocados muito mais em uma ordem política, do que das prioridades em saúde ou da qualidade assistencial. Mais importante que o financiamento, por valer ainda mais que o dinheiro, temos um fluxo de informação muito restrito e insuficiente. Não há informatização em grande parte do sistema, e onde há, ela não é integrada com os demais pontos assistenciais.

Deveríamos ter um sistema centrado nas pessoas, em que a trajetória clínica delas fosse a mais curta possível, sem barreiras de acesso, e na qual a informação e o dinheiro seguissem o paciente a fim de possibilitar e premiar a solução dos problemas de saúde realizada no local mais apropriado, no tempo adequado e com qualidade suficiente. Ao invés disso, temos um labirinto desconhecido de caminhos, onde a pessoa não é acompanhada de suas informações clínicas e onde, muitas vezes, o recurso financeiro não está aplicado no serviço que melhor poderia resolver o seu problema.

Este cenário de fragilidades não deve ser desestimulante, ao contrário, ele oferece uma série de oportunidades de atuação que podem rapidamente trazer muito mais funcionalidade ao SUS, com seu consequente efeito sobre a melhora da saúde das pessoas. Mas, para isso, é necessário enfrentar o fluxo desordenado das pessoas, das informações clínicas e do financiamento, além das pressões da burocracia que não quer abrir mão do seu papel planificador central, da pressão das corporações, que põem seus interesses particulares à frente do interesse dos cidadãos, e das pressões dos maus políticos, que se beneficiam do ambiente de desorganização para reforçarem seu papel clientelista.

Até aqui, exploramos uma visão sistêmica do SUS. A partir de agora, essa visão sistêmica se aprofundará sobre os aspectos da organização da APS brasileira.

\section{A organização da atenção primária brasileira}

AAPS brasileira se caracteriza por um grande número de Unidades de Saúde, com coexistência do modelo Estratégia Saúde da Família (ESF) e arranjos organizacionais heterogêneos. No final de 2019, havia quase 45 mil Unidades Básicas de Saúde, com 43.458 equipes de ESF com cobertura potencial de cerca de 150 milhões de pessoas. Todas as equipes de ESF usam o Sistema de Informação em Saúde para a Atenção Básica (SISAB) como sistema de informação. Seu acesso se dá por uso de fichas em papel (CDS), uso do prontuário eletrônico eSUS-AB ou uso de outro prontuário eletrônico com transmissão de dados via estratégia chamada "thrift". 
Em abril de 2019, ao se analisar os dados do SISAB, havia cerca de 90 milhões de pessoas cadastradas na ESF sem contar pessoas duplicadas. Uma diferença, portanto, de quase 60 milhões de pessoas vivendo sob as áreas de adscrição (ou de responsabilidade) das equipes, mas que nem cadastradas estavam! Esse fato corrobora o achado de inúmeras investigações que mostram que o acesso de primeiro contato é o atributo mais frágil da APS brasileira.

O modelo de financiamento vigente até 2019 contribuiu, após a esperada expansão da ESF em meados da década passada, para esta dificuldade em ampliar o número de pessoas sob efetiva responsabilidade das equipes. Esse modelo estava esgotado e apresentava limitações quanto à capacidade de induzir respostas primordiais aos desafios do sistema e da APS, tais como a efetivação do acesso e a produção de melhores resultados em saúde. Ele era formado por vários componentes. O primeiro era composto em parte por uma parcela fixa, calculada a partir do tamanho populacional do município, o chamado "PAB fixo". Este recurso, superior a $\mathrm{R} \$ 4$ bilhões anuais, é repassado sem nenhuma exigência auditável de contrapartida do município, uma vez que não havia registro da vinculação de pessoa a pessoa e nem critério de homogeneidade na fiscalização de sua aplicação na APS pelos órgãos de controle. O segundo componente do modelo de financiamento até então vigente era o chamado "PAB variável", um pout-pourri de incentivos financeiros repassados de acordo com o credenciamento e mínima atividade dos mais diversos tipos de equipes de ESF, Consultório na Rua, equipes prisionais, etc. Para receber o "PAB variável", o município necessitava credenciar a equipe junto a SAPS, manter o cadastro desta equipe no SCNES completo,e informar alguma atividade da equipe, mesmo que fosse apenas um procedimento ao mês. No final da década de 1990 e princípio da década de 2000, esse modelo cumpriu um importante papel inicial de descentralização das ações e serviços ambulatoriais de saúde para municípios que anteriormente não possuíam capacidade instalada, conforme analisaram Costa e Pinto. ${ }^{10}$

Contudo, com o passar das duas últimas décadas (1998-2018), o PAB variável passou a concentrar a maior parte dos recursos federais alocados na APS, apesar de apresentar limitações como o excessivo enfoque na capacidade instalada, no condicionamento do repasse a regras federais pouco flexíveis, além da profusão de programas, sendo 20 tipos diferentes em 2019.

O terceiro componente está relacionado ao incentivo repassado aos municípios para a implantação e manutenção de agentes comunitários de saúde nas equipes de saúde da família. Este incentivo é da ordem de mais de $\mathrm{R} \$ 4$ bilhões anuais, e é atrelado a deveres legais dos gestores em assegurarem pagamento de piso nacional, mas desprovido de vinculação e proporcionalidade ao desempenho alcançado pela estratégia.

O quarto e último componente é o Programa de Melhoria de Acesso e Qualidade (PMAQ), que inovou positivamente em 2011 ao abordar explicitamente a necessidade de ampliar o acesso e de melhorar a qualidade do cuidado na ESF. Apesar deste grande avanço, o PMAQ possuía um método com inúmeras limitações. Um de seus componentes é baseado em coletas presenciais de baixa periodicidade (2 a 3 anos), sem cronograma pré-estabelecido e transparente, usa diversos questionários sem qualquer validação nacional estatística e científica, que geram mais de 1.000 variáveis que compõem a avaliação final de cada equipe de ESF. Quando se pretende medir tudo, não se mede nada. 
Quase $\mathrm{R} \$ 2$ bilhões são repassados por meio da avaliação do PMAQ, apenas para as equipes que aderiam, visto que é uma avaliação opcional que atinge cerca de 36 mil equipes de ESF, cerca de $80 \%$ do total das equipes existentes em 2019. O que significa que para 20\% das ESF não há qualquer tipo de avaliação do cuidado prestado à população brasileira.

Defendemos que essas questões estruturais certamente dificultam um real avanço do acesso e da qualidade na APS brasileira. Apesar de trabalharem sob condições repletas de obstáculos, as equipes de ESF garantiram avanços fundamentais e inquestionáveis. Redução da mortalidade infantil, qualificação do pré-natal, cuidado de condições agudas menos graves, cuidado de condições crônicas, incluindo de saúde mental, demonstrando redução de mortalidade por algumas condições e diminuição de internações sensíveis à APS. 11,12,13,14,15 Entretanto, muitos novos e outros antigos desafios precisam ser superados. A cobertura vacinal vem diminuindo, a qualidade do cuidado de condições crônicas, como diabetes e hipertensão, não mostram avanços semelhantes a outros países de renda média, o número de internações por condições sensíveis à APS ainda é muito alto, quase o dobro de países com sistemas nacionais de saúde baseados em APS, a incidência de sífilis congênita é inaceitável, e a resolutividade clínica, em níveis nacionais, é insuficiente, como bem demonstram as intervenções de telemedicina aplicadas a listas de espera por consulta com especialistas originadas da APS em diversos locais diferentes do país, em especial da região Sul. ${ }^{16,17}$

Contribuem para este cenário, a ausência de profissionais adequadamente formados para trabalhar em APS e a não-incorporação sistemática de ferramentas de governança clínica. ${ }^{18,19,20}$ Atualmente, existem no Brasil apenas 6 mil médicos de família e comunidade. O número de enfermeiros e odontólogos com especialização ou residência em APS é ainda menor. O uso de protocolos clínicos, linhas de cuidado e sistemas de suporte assistencial é muito baixo no país. Além disso, a organização da agenda dos profissionais e das atividades multiprofissionais na maior parte das equipes não privilegia a prática clínicoassistencial. Há pouca subsidiariedade entre os integrantes das equipes, gerando ineficiência e restrição de acesso. Enfermeiros realizam poucas atividades assistenciais, por diversos motivos, entre eles a ausência de protocolos clínicos, legalmente exigidos para tal fim. Médicos e enfermeiros necessitam ter uma prática clínica mais interdependente e de apoio mútuo para ampliar a capacidade e a qualidade do cuidado. Os demais profissionais de saúde de nível superior, apesar de presentes em grande número (são mais de 71 mil), precisam também de maior interação assistencial com o binômio essencial médico-enfermeiro, principalmente apoiados por iniciativas de governança clínica que definam as suas atribuições em nível local e ao longo da trajetória clínica das pessoas.

Para enfrentar essas e outras disfunções da APS do SUS, foram identificados sete desafios principais e para cada um deles uma estratégia foi apresentada (Quadro 1). Ao longo de 2019, as bases para uma reforma da Atenção Primária à Saúde em nível nacional foram identificadas pelo Ministério da Saúde e uma série de mudanças foram propostas, discutidas e realizadas, para que, a partir de 2020 , o Brasil pudesse ter um arcabouço federal e o seguimento de sua implementação. 
Quadro 1. Atenção Primária à Saúde no SUS: desafios e estratégias para enfrentamento.

\begin{tabular}{|c|c|c|}
\hline \# & Desafios & Estratégia/Programas propostos \\
\hline 1 & Falta de priorização política real da APS. & Criação da Secretaria [Nacional] de Atenção Primária à Saúde (SAPS). \\
\hline 2 & $\begin{array}{l}\text { Financiamento da APS insuficiente e } \\
\text { focado na estrutura de serviços. }\end{array}$ & Programa "Previne Brasil": reforma do financiamento da APS. \\
\hline 3 & Obstáculos ao acesso de primeiro contato. & Programa "Saúde na Hora": reforma do acesso na APS. \\
\hline 4 & Insuficiência de profissionais qualificados. & $\begin{array}{l}\text { Programa "Médicos pelo Brasil" (MPB): provimento e formação de Médicos de Família } \\
\text { e Comunidade e incentivos financeiros às residências profissionais em APS. }\end{array}$ \\
\hline 6 & $\begin{array}{l}\text { Fragilidade clínica e necessidade de } \\
\text { ampliação do escopo profissional. }\end{array}$ & $\begin{array}{l}\text { Fortalecimento da clínica e ampliação do escopo profissional: Carteira de Serviços da } \\
\text { APS, Linhas de Cuidado, Protocolos Clínicos de Enfermagem. }\end{array}$ \\
\hline 7 & $\begin{array}{l}\text { Ausência de informação de qualidade para } \\
\text { tomada de decisão clínica e gerencial. }\end{array}$ & Produção de informação de qualidade para tomada de decisão clínica e gerencial. \\
\hline
\end{tabular}

Fonte: Elaboração dos autores.

\section{Estratégia \#1: criação da Secretaria de Atenção Primária à Saúde (SAPS)}

Ao longo dos últimos 25 anos, desde a criação do então "Programa de Saúde da Família" (PSF) em 1994, pelo Ministério da Saúde, nunca tinha havido uma Secretaria específica para a gestão da APS a nível federal; o que existia anteriormente era um Departamento vinculado a uma Secretaria que também cuidava da atenção especializada e hospitalar. Para enfrentar essa falta de priorização política, de fato, em maio de 2019, foi realizada uma reforma administrativa no Ministério da Saúde e através do Decreto n 9.795 , de 17 de maio de 2019, foi criada a Secretaria de Atenção Primária à Saúde (SAPS). ${ }^{3,21}$ A partir da mudança estrutural, os projetos, as demandas e a necessidade por recursos financeiros para a APS ganharam voz e igualdade de condições na disputa saudável e competitiva com as demais Secretarias. Além disso, o Ministério da Saúde publicou após 100 dias de gestão, ${ }^{22}$ que a estratégia número 1 da gestão 2019-2022 contemplaria a coordenação de estratégias e projetos referentes à APS, oferecendo as condições e o apoio necessário para que elas tivessem êxito. A partir daí as principais ações do Ministério da Saúde no ano de 2019 corresponderam à APS.

Outro ganho com a criação da SAPS, foi a criação de dois departamentos: o Departamento de Saúde da Família (DESF) e o Departamento de Promoção de Saúde (DEPROS). O primeiro foi reforçado e passou a ser responsável por expandir em número e qualidade as equipes de APS, contando com uma coordenação responsável pela qualificação da informação, outra com responsabilidade exclusiva em analisar e propor o financiamento da APS brasileira e uma terceira cuja linha de trabalho é garantir a presença forte dos atributos essenciais e derivados da APS.

O segundo, o DEPROS, tinha como objetivo integrar todas ações de promoção de saúde do Ministério, além de propor a sistematização do cuidado assistencial das condições crônicas fortemente ligado às ações de promoção e prevenção. O foco maior das ações de promoção são aquelas ligadas à alimentação saudável, à prática de atividade física e à integração entre as ações de saúde com a escola e com as políticas sociais do Governo.

Outro Departamento que integra a estrutura da Secretaria é o Departamento de Ações Programáticas Estratégicas (DAPES), departamento que já compunha o Ministério da Saúde, e cuja atribuição precípua é a gestão de políticas de saúde para populações naquilo que se convencionou chamar de "ciclos de vida", como crianças, adolescentes, mulheres, homens e pessoas idosas. Ao integrar a SAPS, o desafio para esse 
Departamento passa a ser compatibilizar o advocacy feito pela oferta de atenção adequada a tais segmentos junto aos atores envolvidos - cidadãos, organizações da sociedade civil, Ministério Público, poder judiciário e legislativo - com a articulação com as equipes de atenção primária e demais serviços da rede de atenção para a efetivação de respostas em saúde para tais segmentos que acabam por repercutir sobre o conjunto de agravos prioritários da APS.

\section{Estratégia \#2: Programa Previne Brasil}

O Programa "Previne Brasil", ${ }^{23}$ que instituiu o novo financiamento da APS, pretende corrigir o financiamento insuficiente e destinar o aumento do investimento da APS às equipes e municípios que mais e melhor trabalham nas equipes de Saúde da Família. Para o êxito da nova proposta, foram adicionados $\mathrm{R} \$ 2$ bilhões ao volume de repasses federais aos municípios no ano de 2020 em comparação ao ano de 2019. Além disso, criamos as equipes de Atenção Primária à Saúde (eAPS) a fim de possibilitar crescimento no número de equipes que recebem repasses financeiros federais, com menor exigência de carga horária dos profissionais médicos, enfermeiros e dentistas, dando condições aos municípios de criarem equipes reconhecidas e acompanhadas pelo Governo Federal sem a contratação de profissionais adicionais. Reconhecemos, desta forma, as inciativas e o protagonismo dos municípios em ofertar ações e serviços de APS aos seus cidadãos.

Paralelamente à criação das eAPS, mantivemos e fortalecemos a Estratégia Saúde da Família com o status de estratégia prioritária para a APS brasileira. Os municípios que reduzirem número de ESF não poderão credenciar eAPS. Além disso, os incentivos destinados às residências de medicina de família e comunidade, de enfermagem de família e comunidade e de odontologia, assim como a alocação de médicos do Programa Médicos pelo Brasil, só são destinados às equipes de ESF.

O "Previne Brasil" é um modelo misto de financiamento, como os utilizados pelos melhores sistemas de saúde do mundo, ${ }^{24,25,26,27}$ especialmente dos países ligados à Organização para a Cooperação e Desenvolvimento Econômico (OCDE), em que cada componente traz equilíbrio ao integrar-se com os demais. O primeiro e principal componente é o da capitação ponderada, responsável por cerca de $50 \%$ do investimento federal em APS. Estabelece um valor anual por pessoa sob responsabilidade das equipes de Saúde da Família ou de Atenção Primária, ponderado por variáveis de vulnerabilidade individual: extremos de idade ( $<5$ ou >65 anos), ou beneficiários do Bolsa Família, do Benefício de Prestação Continuada e de benefício previdenciário de até dois salários mínimos. Além da ponderação em nível individual, há uma ponderação relativa ao contexto de determinação do processo de adoecimento. Cidades pequenas e remotas recebem maior valor que cidades intermediárias. E essas últimas, maior valor per capita que os centros urbanos, de acordo com a classificação técnica do IBGE que será utilizada no Censo Demográfico de $2020 .{ }^{28}$

O segundo componente é o do pagamento por resultados. Vinte e um indicadores de saúde e indicadores globais da qualidade da APS serão monitorados mensalmente. ${ }^{29}$ As equipes e municípios receberão recurso adicional de acordo com seu desempenho no atingimento das metas. Os indicadores relacionados a condições de saúde versam sobre saúde infantil, saúde da mulher, condições crônicas, saúde mental, infecções sexualmente transmissíveis e internações sensíveis à atenção primária. 
Os indicadores globais são relacionados à fidelização das pessoas às equipes (Net Promoter Score), ${ }^{30}$ qualidade da relação médico-paciente (PDQR9) ${ }^{31}$ e presença e extensão dos atributos da APS (PCAToolBrasil). ${ }^{32}$ Todos esses escores oriundos desses indicadores globais são medidos por meio de instrumentos cientificamente definidos e validados no Brasil, ${ }^{30,31,32}$ permitindo uma avaliação local e regional ampla da APS e inúmeras comparações, inclusive no cenário internacional.

O terceiro e último componente - o componente dos incentivos - é relacionado às ações estratégicas e ao cuidado de populações especificamente vulneráveis. Os incentivos estratégicos são relacionados à ampliação do acesso e do horário de atendimento (Saúde na Hora), ao Programa de Residência Médica e Multiprofissional (considerados padrão-ouro para a formação de profissionais especialistas no Brasil), à informatização (Conecte-SUS e Informatiza-APS), às ações de promoção e prevenção (Saúde na Escola, Academia da Saúde), entre outros. Os incentivos relacionados às populações vulneráveis incluem a população ribeirinha e amazônica, a população em situação de rua, adolescentes em medidas socioeducativas, etc.

A fim de manter a priorização imprescindível da APS para alcançar a funcionalidade do SUS, nos próximos anos deve-se destinar mais recursos financeiros para o componente da capitação ponderada e do pagamento por desempenho. Neste último, só devem ser acrescentados novos indicadores se o valor orçamentário absoluto destinado a este componente crescer. Na mesma medida, o ideal seria que o valor da capitação crescesse em termos absolutos e relativos. Em valores absolutos, até que a soma dos repasses federais destinados a APS alcançasse cerca de $50 \%$ do custo total de investimento e manutenção das estratégias de APS, especialmente das equipes de Saúde da Família. Em termos relativos, o valor da capitação deve atingir $60 \%$ do total de repasses federais. Hoje, ele está em torno de $50 \%$.

Além de remodelar o financiamento, garantindo mais e melhores resultados na APS brasileira, outro objetivo do Previne Brasil é alcançar até 2022 a marca de $80 \%$ de cobertura real, isto é, cerca de 170 milhões de pessoas cadastradas, sem os milhões de cadastros duplicados existentes até 2019. Para tanto, serão necessárias 50.000 equipes de Saúde da Família existentes e financiadas. Em dezembro de 2018, tínhamos 42.975 ESF financiadas. Ao final de 2019, em dezembro, tínhamos 43.458 ESF em financiamento. Ao virar o ano, o primeiro pagamento referente a CNES (janeiro de 2020), que marcará o início da Reforma apresentada pelo Previne Brasil, irá contemplar 45.796 ESF financiadas. A essas, se somarão 1.192 equipes de Atenção Primária (eAPS) credenciadas e financiada desde dezembro/2019. Como cada ESF tem cobertura potencial igual a 2 equipes de eAPS, iniciaremos o ano com 46.392 "ESF equivalentes" sendo financiadas. A meta para o final do ano de 2020 é de 46.600 ESF equivalentes financiadas.

Além da forte expansão da ESF, o Previne Brasil traz uma outra novidade imprescindível para tornar o SUS um sistema de saúde realmente centrado nas pessoas. A Organização para Cooperação e Desenvolvimento Econômico (OCDE) estipulou que uma das características essenciais de um sistema de saúde centrado nas pessoas é a livre escolha do provedor de APS. ${ }^{33}$ Como o livre-arbítrio é princípio inerente e fundamental de uma democracia liberal, a definição política proposta pelo Ministério da Economia está presente no componente de capitação ponderada. Quanto melhor for o trabalho de determinada equipe de Saúde da Família, mais procurada ela será, e mais fácil será o alcance dos parâmetros de cadastramento, fato que gerará um processo de competição virtuosa por mais pessoas vinculadas às equipes, especialmente as mais vulneráveis. 
Esta possibilidade de livre escolha da Unidade de Saúde (ou provedor de APS) pode ou não ser definida em nível municipal, mas perder a oportunidade de centrar o sistema nas pessoas e introduzir mecanismo de competição de escolha de provedor pela qualidade de atendimento não parece uma escolha razoável. Ainda mais com o avanço das Tecnologias de Informação e Comunicação (TIC) que permitem que o trabalho assistencial seja intensamente conectado à abordagem territorial realizada por toda a equipe e, especialmente, pelos Agentes Comunitários de Saúde e pela possibilidade trazida pelo Decreto $n^{\circ} 9.723$, de 11 de março de 2019, de se realizar a gestão de cadastros duplicados com maior eficiência, na medida em que o próprio Ministério da Economia através da Receita Federal realiza periodicamente a gestão e atualização centralizada dos CPFs válidos e inválidos. Contar o número de pessoas sem duplicá-las, é o elemento central para se ter o denominador de uma série de indicadores de saúde na atenção primária, evitando-se o desperdício de recursos públicos.

\section{Estratégia \#3: Saúde na hora}

A fim de enfrentar a dificuldade de acesso às Unidades de Saúde, aliada à estratégia de aumentar a escala e a carteira de serviços da APS - que será abordada mais adiante -, foi lançado também em maio de 2019 o Programa "Saúde na Hora". ${ }^{34}$ Este programa já trazia os fundamentos do Previne Brasil: mais recursos financeiros para a APS, exigência de informatização, além de flexibilidade e autonomia para o gestor, assim como o livre acesso das pessoas durante o horário estendido. Em seus dois modelos propostos, o Saúde na Hora dobra o financiamento das Unidades de Saúde que ficam abertas 60 ou 75 horas por semana, isto é, de 12 a 15 horas ininterruptas por dia útil. Lançado com meta de credenciar 1.000 Unidades de Saúde até dezembro/2019, o Saúde na Hora atingiu o número de 1.564 Unidades de Saúde da Família credenciadas nesse período. Essas mais de 1.500 USF representam 5.900 ESF e 2.085 equipes de Saúde Bucal, beneficiando diretamente mais de 20 milhões de pessoas.

\section{Estratégia \#4: Programa Médicos pelo Brasil}

A insuficiência de profissionais com formação específica para trabalhar em Atenção Primária não é um desafio exclusivo do Brasil. Diversos outros países passam ou passaram pelo mesmo problema. Esse problema torna-se crônico se enfrentado apenas com estratégias emergenciais, ou com alto potencial de deterioração da qualidade clínica, se o método de formação não for regido pelos atributos da APS, nem acompanhado por processo rigoroso de monitoramento e avaliação.

Em nosso país, a insuficiência no número de médicos trabalhando em APS se destaca. Apesar do Programa Mais Médicos ter enfrentado emergencialmente este problema, sua fragilidade normativa e seus escassos mecanismos de gestão e avaliação atenuaram o problema, mas não o resolveram. A fim de superar essas limitações, lançamos por meio de Medida Provisória o Programa "Médicos pelo Brasil". Após exatos 120 dias de exaustiva discussão no Congresso Nacional, o Médicos pelo Brasil foi aprovado por 391 votos a favor e 6 votos contrários em votação nominal na Câmara. ${ }^{35} \mathrm{O}$ que impressiona nesse processo, aprovado também no Senado, é que tanto o componente de provimento como o componente de formação de médicos de família e comunidade praticamente não sofreram nenhuma alteração em relação à proposta original do Ministério da Saúde. 
O Médicos pelo Brasil alia um método perene de provimento médico por meio de contratação federal de médicos via Agência para o Desenvolvimento da Atenção Primaria à Saúde (ADAPS), instituição privada sem fins lucrativos e que, portanto, contratará seus trabalhadores pelo regime da Consolidação das Leis do Trabalho (CLT) a um processo de formação em larga escala e com qualidade de Médicos de Família e Comunidade. A contratação de médicos pela ADAPS permitirá que apenas médicos certificados pelo Conselho Federal de Medicina (CFM) possam ser contratados após processo seletivo público que privilegiará o mérito como critério de seleção. Médicos especialistas em Medicina de Família e Comunidade ou Clínica Médica ingressarão no formato CLT como tutores. Médicos não-especialistas ingressarão como bolsistas num curso de especialização em Medicina de Família e Comunidade com dois anos de duração e 60 horas semanais, sob supervisão da Sociedade Brasileira de Medicina de Família e Comunidade (SBMFC). Todos os médicos participantes do Programa receberão gratificação de acordo com as características dos municípios, assim como receberão gratificação por desempenho que variará de acordo o nível de progressão na contratação CLT. ${ }^{22,35} \mathrm{O}$ desempenho dos médicos será medido com o mesmo conjunto de indicadores do Previne Brasil, fazendo com que gestores, equipes e os Médicos pelo Brasil tenham a mesma missão de melhoria da qualidade da APS brasileira.

Além do Médicos pelo Brasil, há novos estruturantes investimentos financeiros na residência em Medicina de Família e Comunidade, na residência em Enfermagem de Família e Comunidade e na residência em Odontologia em Saúde da Família. ${ }^{36}$ Municípios que compuserem equipes de Saúde da Família com um ou mais residentes dessas três categorias profissionais, em residências aprovadas pela Comissão Nacional de Residência Médica ou Comissão Nacional de Residência Multiprofissional em Saúde, receberão incentivos financeiros a partir de março de 2020. Estes objetivam propiciar melhores condições de formação aos profissionais de nível superior essenciais para a qualificação da APS brasileira.

\section{Estratégia \#5: Conecte-SUS e Informatiza-APS}

Sem um fluxo objetivo de informações clínicas essenciais que acompanhem a trajetória clínicoassistencial das pessoas, a efetividade de um sistema universal de saúde fica limitada, gerando riscos à saúde das pessoas e à sustentabilidade do sistema. Para garantir este fluxo de informações no SUS, o Ministério da Saúde criou o Conecte-SUS. Trata-se de uma estratégia de integração de dados clínicos e administrativos entre os sistemas de informação existentes a partir da identificação unívoca das pessoas por meio do CPF. No primeiro momento, está prevista a integração das informações clínicas da APS (SISAB), administrativas ambulatoriais (SIA), hospitalares $(\mathrm{SIH})$ e de procedimentos de alta complexidade (APAC), assim como informações sobre imunização (SIPNI) e medicamentos (BENAFAR). Essas informações estarão disponíveis em um visualizador clínico com acesso confidencial, seguro e adequado a cada categoria profissional. No momento, o Conecte-SUS está estruturado em fase piloto no Estado do Alagoas. ${ }^{37}$

A fim de dar maior potência ao Conecte-SUS é imprescindível que o grau de informatização e de uso de prontuário eletrônico na APS seja maior que o atual. Em meados de 2019 , cerca de $60 \%$ das equipes da ESF transmitiam informações ao SISAB via prontuário eletrônico. Frente a essa evolução, construída autônoma e independentemente pelos municípios e com grandes avanços foi criado pelo Ministério da Saúde, o Programa "Informatiza-APS". ${ }^{38}$ 
O Informatiza-APS é uma estratégia de estímulo à informatização e uso do prontuário eletrônico na APS. O Ministério da Saúde repassa, desde novembro de 2019, incentivos financeiros de custeio mensal aos municípios cujas equipes de Saúde da Família enviem seus dados assistenciais em um padrão de quantidade e qualidade pré-estabelecido via integrador "thrift" do SISAB. Com essa estratégia, que inclui incentivo financeiro de implantação para aqueles municípios ainda não informatizados, estabeleceu-se como meta que $90 \%$ das equipes de Saúde da Família enviem seus dados via prontuário eletrônico até o final de 2022.

Essa estratégia possibilita total liberdade aos municípios escolherem entre a estratégia eSUS-AB ou outro sistema de sua preferência. A SAPS apoia os municípios que optarem pelo eSUS-AB, ao implantar pela primeira vez um serviço de helpdesk do mesmo. Caso a gestão municipal opte livremente por outro sistema, a SAPS disponibiliza desde a lista de hardware necessária, com especificação mínima para êxito, assim como um termo de referência default para que o município possa fazer um processo licitatório com mais segurança.

\section{Estratégia \#6: fortalecimento da clínica e ampliação do escopo profissional, carteira de serviços da APS}

Para o avanço na qualidade assistencial, as decisões clínicas devem ser tomadas a partir das melhores evidências cientificas disponíveis, adaptadas ao SUS, e com subsidiariedade tanto entre integrantes das equipes de APS, como entre os diversos pontos assistenciais da Rede de Atenção à Saúde. A iniciativa escolhida para essa qualificação foi a criação de 22 linhas de cuidado que definam a trajetória clínica mais adequada para que as pessoas obtenham o cuidado certo, no lugar certo, no tempo certo com o melhor resultado possível.

Para tanto, o Ministério da Saúde estabeleceu uma parceria com o Instituto de Avaliação de Tecnologia em Saúde (IATS) do Hospital de Clínicas de Porto Alegre (HCPA). O processo de construção das linhas de cuidado partirá do conjunto da produção semelhante já desenvolvida anteriormente em nível federal, estadual ou municipal. Além disso, esforço adicional foi dirigido para que o modelo de apresentação das linhas seja sucinto e de fácil entendimento, baseado quase exclusivamente em fluxogramas clínicos. As passagens das pessoas de um ponto assistencial a outro incorporam os Protocolos de Encaminhamento do próprio Ministério da Saúde.

A primeira linha de cuidado, Manejo do Acidente Vascular Cerebral no Adulto, foi colocada em consulta pública no final de dezembro de 2019. Desde então e até meados de 2021, será disponibilizada mensalmente uma linha de cuidado até se alcançar a meta de 22 linhas.

Definir a melhor trajetória clínica com base nas melhores evidências é fundamental, mas não será a única estratégia para fortalecer a qualidade clínica na APS. A fim de ampliar o escopo profissional da enfermagem, Protocolos de Enfermagem serão publicados pelo MS acerca dos principais temas assistenciais que exigem maior integração entre médicos e enfermeiros. Para tanto, os protocolos, após definição de sua primeira versão, serão enviados para o Conselho Federal de Enfermagem e Conselho Federal de Medicina para avaliação, sendo publicados em acordo entre os dois Conselhos e o Ministério da Saúde.

Outras ferramentas de suporte multiprofissional, como o sistema de suporte clínico do 0800644 6543, que foi ampliado, serão ofertadas ao longo do ano de 2020. 
Já a carteira de serviços para a atenção primária à saúde (CaSAPS) ficou em consulta em agosto de 2019 e foi lançada em dezembro do mesmo ano, ${ }^{20}$ com contribuições da Sociedade Brasileira de Medicina de Família e Comunidade (SBMFC), da Associação Brasileira de Enfermagem de Família e Comunidade (ABEFACO) e da Associação Brasileira de Odontologia (ABO). O documento teve também a participação do Conselho Nacional de Secretarias Municipais de Saúde (CONASEMS) e do Conselho Nacional de Secretários de Saúde (CONASS), além de diversos profissionais de saúde que enviaram sugestões à consulta pública.

\section{Estratégia \#7: produção de informação de qualidade para tomada de decisão clínica e gerencial}

A fim de disponibilizar para todos níveis de gestão, assim como para a prática assistencial em toda Rede de Atenção e para a produção de conhecimento, informação de qualidade será produzida com intensidade nos próximos anos. A fim de qualificar e homogeneizar as informações disponibilizadas pelo Ministério diversas ações estão em andamento.

O Conecte-SUS é a principal delas. Entretanto, a fim de captar informações que não circulam por dentro dos sistemas de informação do SUS, o Ministério da Saúde firmou em dezembro de 2019 um termo de cooperação com IBGE, ${ }^{39}$ a fim de ampliar o componente de atenção primária à saúde nas pesquisas administrativas e domiciliares de base demográfica já existentes. Trata-se de um resgate histórico do IBGE como grande e maior avaliador externo das Políticas Públicas em Saúde no Brasil, com recursos perenes alocados para os próximos quatro anos.

Dessa forma, a Pesquisa Nacional de Saúde (PNS), a Pesquisa Nacional de Amostragem por Domicílios Contínua (PNAD), a Pesquisa Nacional de Demografia e Saúde (PNDS) e a pesquisa sobre Assistência Médico-Sanitária (AMS) incluirão módulos específicos sobre atenção primária à saúde e desenvolvimento infantil relevantes para as principais políticas do Ministério da Saúde.

Este conjunto de perguntas contemplará, sempre que possível, instrumentos de pesquisa validados nacional e internacionalmente a fim de permitir comparações longitudinais e também com outros países. Os principais temas incorporados até então foram, a avaliação da APS, por meio do uso de PCATool-Brasil em suas múltiplas versões, o PDQR9 para medir a qualidade da relação médico-paciente, e o Primeira Infância para Adultos Saudáveis (PIPAS) para avaliar o desenvolvimento infantil na primeira infância.

Em nova cooperação com o IBGE a ser celebrada em 2020, a linkagem desses dados demográficos com os dados administrativos dos sistemas de informação será possível. A partir dessa linkagem, as bases de dados serão abertas a toda comunidade científica a fim de promover maior produção de pesquisa de qualidade, e também, inteligência interinstitucional na era do big data.

Além da cooperação com IBGE, pela primeira vez na história do Ministério da Saúde, foi estabelecido um acordo com a Organização para Cooperação e Desenvolvimento Econômico (OCDE). O Ministério iniciou sua representação formal junto a OCDE em 2019 e, a partir de então, estabeleceu um acordo para a realização de duas grandes avaliações nacionais a ser realizada pelo time de pesquisadores ligados à instituição internacional. Uma delas será sobre todo o SUS e a outra será especifica sobre APS no SUS. Na mesma linha de ampliar as cooperações e comparações internacionais, foi aprovada uma Carta Consulta do Ministério da Saúde com o Banco Mundial. Técnicos e consultores do Banco, por meio de nova linha de crédito, contribuirão para fortalecer o Programa "Previne Brasil", tendo como um dos objetivos a qualificação da informação para a tomada de decisão. 
Esses sete desafios e as estratégias para enfrentá-los representam mudanças estruturantes e investimentos em governança clínica para transformar a APS brasileira, garantindo mais e melhor saúde para a população, com mecanismos transparentes e técnicos para sua medição. A forma proposta de monitoramento se dará através de dashboard publicado mensalmente junto ao site da SAPS, na funcionalidade intitulada eGestor-APS.

Eles não são suficientes para que todas as disfunções do SUS sejam resolvidas, mas certamente são os mais prementes e fundamentais para uma APS forte, resolutiva e melhor acesso de primeiro contato. Dessa forma, teremos condições para fortalecer o SUS, melhorando a saúde das pessoas, com equidade e dignidade. A partir da consolidação do fortalecimento da APS será premente rever e reformar tanto o financiamento, como os aspectos de governança clínica, da atenção especializada e hospitalar.

\section{REFERÊNCIAS}

1. Starfield B, Shi L, Macinko J. Contribution of primary care to health systems and health. Milbank Q. 2005;83(3):457-502.

2. Holland M. Fiscal crisis in Brazil: causes and remedy. Brazil J Polit Econ. 2019 Mar;39(1):88-107. DOI: https://dx.doi.org/10.1590/010135172019-2918

3. Decreto no 9.795, de 17 de maio de 2019 (BR). Aprova a Estrutura Regimental e o Quadro Demonstrativo dos Cargos em Comissão e das Funções de Confiança do Ministério da Saúde, remaneja cargos em comissão e funções de confiança, transforma funções de confiança e substitui cargos em comissão do Grupo-Direção e Assessoramento Superiores - DAS por Funções Comissionadas do Poder Executivo - FCPE. Diário Oficial da União, Brasília (DF), 2019 mai 17: Seção 1.

4. Ferrer APS. Avaliação da atenção primária à saúde prestada a crianças e adolescentes na região oeste do município de São Paulo [tese]. São Paulo (SP): Universidade de São Paulo - Faculdade de Medicina; 2013.

5. Harzheim E, Pinto LF, Hauser L, Soranz D. Avaliação dos usuários crianças e adultos quanto ao grau de orientação para Atenção Primária à Saúde na cidade do Rio de Janeiro, Brasil. Ciênc Saúde Coletiva. 2016;21(5):1399-408.

6. D’Avila OP, Pinto LFS, Hauser L, Gonçalves MR, Harzheim E. O uso do Primary Care Assessment Tool (PCAT): uma revisão integrativa e proposta de atualização. Ciênc Saúde Coletiva. 2017 Mar;22(3):855-65.

7. Iser BPM. Prevalência de diabetes autorreferido no Brasil: prevalência atual e tendências [tese]. Porto Alegre (RS): Universidade Federal do Rio Grande do Sul (UFRGS) - Programa de Pós-Graduação em Epidemiologia; 2016.

8. Tortorella CCS, Corto ACT, Gonzáles-Chica DA, Melhen ARF. Tendência temporal da prevalência de hipertensão arterial sistêmica e diabetes mellitus entre adultos cadastrados no Sistema Único de Saúde em Florianópolis, Santa Catarina, 2004-2011. Epidemiol Serv Saúde. 2017 Jul/Set;26(3):469-80.

9. Global Burden of Diseases (GBD) Collaborators. Burden of disease in Brazil, 1990-2016: a systematic subnational analysis for the Global Burden of Disease Study 2016. Lancet. 2018 Sep;392(10149):760-75. DOI: https://doi.org/10.1016/S0140-6736(18)31221-2

10. Costa NR, Pinto LF. Avaliação de programa de atenção à saúde: incentivo à oferta de atenção ambulatorial e a experiência da descentralização no Brasil. Ciênc Saúde Coletiva [Internet]. 2002; [citado 2020 jan 19]; 7(4):907-23. Disponível em: http://www.scielo.br/pdf/ csc/v7n4/14614.pdf DOI: http://dx.doi.org/10.1590/S1413-81232002000400022

11. Macinko J, Souza MFM, Guanais FC, Simões CCS. Going to scale with community-based primary care: an analysis of the family health program and infant mortality in Brazil, 1999-2004. Soc Sci Med. 2007 Nov;65(10):2070-80.

12. Bezerra Filho JG, Kerr LRFS, Miná DL, Barreto ML. Spatial distribution of the infant mortality rate and its principal determinants in the State of Ceará, Brazil, 2000-2002. Cad Saúde Pública. 2007 May;23(5):1173-85.

13. Piccini RX, Facchini LA, Tomasi E, Thaumé E, Silveira DS, Siqueira FV, et al. Effectiveness of antenatal and well-baby care in primary health services from Brazilian South and Northeast regions. Rev Bras Saúde Matern Infant. 2007 Mar;7(1):75-82.

14. Aquino R, Oliveira NF, Barreto ML. Impact of the family health program on infant mortality in brazilian municipalities. Am J Public Health. 2009 Jan;99(1):87-93. 
15. Pinto LF, Giovanella L. Do Programa à Estratégia Saúde da Família: expansão do acesso e redução das internações por condições sensíveis à atenção básica (ICSAB). Ciênc Saúde Coletiva [Internet]. 2018 Jun; [citado 2020 feb 03]; 23(6):1903-14. Available from: http://www.scielo.br/scielo.php?script=sci_arttext\&pid=S1413-81232018000601903\&lng=en DOI: http://dx.doi.org/10.1590/141381232018236.05592018

16. Katz N, Roman R, Rados DV, Oliveira EB, Schmitz CAA, Gonçalves MR, et al. Acesso e regulação ao cuidado especializado no Rio Grande do Sul: a estratégia RegulaSUS do TelessaúdeRS-UFRGS. Ciênc Saúde Coletiva [Internet]. 2019 Out; [citado 2010 fev 03] Disponível em: http://www.cienciaesaudecoletiva.com.br/artigos/acesso-e-regulacao-ao-cuidado-especializado-no-rio-grande-do-sul-a-estrategia-regulasus-do-telessaudersufrgs/17400?id=17400

17. Zanotto BS, Etges APBS, Siqueira AC, Silva RS, Bastos C, Araújo AL, et al. Avaliação econômica de um serviço de telemedicina para ampliação da Atenção Primária à Saúde no Rio Grande do Sul: o microcusteio do Projeto TeleOftalmo. Ciênc Saúde Coletiva [Internet]. 2019 Out; [citado 2020 fev 03]. Disponível em: http://www.cienciaesaudecoletiva.com.br/artigos/avaliacao-economica-de-um-servico-de-telemedicina-para-ampliacao-da-atencao-primaria-a-saude-no-rio-grande-do-sul-o-microcusteio-do-projeto-teleoftalmo/17394

18. Ferreira RC, Fiorini VML, Crivelaro E. Formação profissional no SUS: o papel da Atenção Básica em Saúde na perspectiva docente. Rev Bras Educ Med. 2010 Jun;34(2):207-15. DOI: https://dx.doi.org/10.1590/S0100-55022010000200004

19. Janett R, Yeracaris P. Electronic medical records in the American Health System: challenges and lessons learned. Ciên Saúde Coletiva [Internet]. 2019 Out; [citado 2020 fev 03]. Disponível em: http://www.cienciaesaudecoletiva.com.br/artigos/electronic-medical-records-in-the-american-health-system-challenges-and-lessons-learned/17396

20. Cunha CRH, Harzheim E, Medeiros OL, D’Ávila O, Wollmann L, Martins C, et al. Carteira de serviços da atenção primária à saúde: garantia de integralidade nas equipes de saúde da família e saúde bucal no Brasil. Ciên Saúde Coletiva [Internet]. 2019 Dez; [citado 2020 fev 03]. Disponível em: http://www.cienciaesaudecoletiva.com.br/artigos/carteira-de-servicos-da-atencao-primaria-a-saude-garantia-de-integralidade-nas-equipes-de-saude-da-familia-e-saude-bucal-no-brasil/17480

21. Reis JG, Harzheim E, Nachif MCA, Freitas JCA, Otávio H, Martins L, et al. Criação da Secretaria de Atenção Primária à Saúde e suas implicações para o SUS. Ciênc Saude Coletiva [Internet]. 2019;24(9):3457-3462.

22. Brasil. Decreto-Lei ${ }^{\circ} 10.283$ de 20 de março de 2020. Institui o Serviço Social Autônomo denominado Agência para o Desenvolvimento da Atenção Primária à Saúde - ADAPS. [acesso em 2020 mar 22] Disponível em: http://www.planalto.gov.br/ccivil_03/_ato2019-2022/2020/ decreto/D10283.htm]

23. Ministério da Saúde (BR). Portaria no 2.979, de 12 de novembro de 2019. Institui o Programa Previne Brasil, que estabelece novo modelo de financiamento de custeio da Atenção Primária à Saúde no âmbito do Sistema Único de Saúde, por meio da alteração da Portaria de Consolidação no 6/GM/MS, de 28 de setembro de 2017. Diário Oficial da União, Brasília (DF), 2019 nov 13: Edição $220:$ Seção 1: 97.

24. Martin J, Associates Limited. Evaluation of the PHO performance programme: final report. Auckland, NZ: Martin, Jenkins and Associates Limited; 2008.

25. Buck D, Dixon A. Improving the allocation of health resources in England: how to decide who gets what. Ideas that change Health Care. London, UK: The Kings Fund; 2013.

26. Kringos DS, Boerma W, van der Zee J, Groenewegen P. Europe's Strong Primary Care Systems are linked to better population health but also to higher health spending. Health Affairs. 2013 Apr;32(4). DOI: https://doi.org/10.1377/hlthaff.2012.1242

27. Chasing C, Chi YL, Smith P, Borowitz M, Thomson S. Paying for performance in health care - implications for health system performance and accountability. Belgium: European Observatory on Health Systems and Policies; 2014.

28. Instituto Brasileiro de Geografia e Estatística (IBGE). Classificação e caracterização dos espaços rurais e urbanos do Brasil: uma primeira aproximação. Rio de Janeiro (RJ): IBGE; 2017.

29. Ministério da Saúde (BR). Portaria no 3.222, de 10 de dezembro de 2019. Dispõe sobre os indicadores do pagamento por desempenho, no âmbito do Programa Previne Brasil. Diário Oficial da União, Brasília (DF), 2019 dez 11: Edição 239: Seção 1: 172.

30. Endeavor Brasil. Net Promoter Score (NPS): como as maiores empresas do mundo medem a satisfação de seus clientes. São Paulo: Endeavor Brasil; 2003; [acesso em 2020 jan 19]. Disponível em: https://endeavor.org.br/estrategia-e-gestao/nps/

31. Wollmann L. Avaliação da relação médico-paciente: tradução e validação do Patient-Doctor Relationship Questionnaire (PDRQ-9) no Brasil [dissertação]. Porto Alegre (RS): Universidade Federal do Rio Grande do Sul (UFRGS) - Pós-Graduação em Epidemiologia;2017.

32. Ministério da Saúde (BR). Secretaria de Atenção à Saúde. Departamento de Atenção Básica. Manual do instrumento de avaliação da atenção primária à saúde: primary care assessment tool PCATool - Brasil. Série A. Normas e Manuais Técnicos. Brasília (DF): Ministério da Saúde; 2010; [acesso em 2020 jan 19]. Disponível em: http://bvsms.saude.gov.br/bvs/publicacoes/manual_avaliacao_pcatool_brasil.pdf 
33. Organisation for Economic Co-Operation and Development (OECD). The OECD Health Project. Towards high-performing health systems. Summary report. Genebra: OECD; 2004; [acesso em 2020 jan 10]. Disponível em: https://www.oecd.org/els/health-systems/31785551.pdf

34. Ministério da Saúde (BR). Portaria no 930, de 15 de maio de 2019. Institui o Programa "Saúde na Hora", que dispõe sobre o horário estendido de funcionamento das Unidades de Saúde da Família, altera a Portaria no 2.436/GM/MS, de 2017, a Portaria de Consolidação nº 2/GM/MS, de 2017, a Portaria de Consolidação nº 6/GM/MS, de 2017, e dá outras providências. Diário Oficial da União, Brasília (DF), 2019 mai 17: Edição 94: Seção 1: 122.

35. Lei no 13.958, de 18 de dezembro de 2019 (BR). Institui o Programa Médicos pelo Brasil, no âmbito da atenção primária à saúde no Sistema Único de Saúde (SUS), e autoriza o Poder Executivo federal a instituir o serviço social autônomo denominado Agência para o Desenvolvimento da Atenção Primária à Saúde (ADAPS). Brasília (DF): Ministério da Saúde; 2019; [acesso em 2020 jan 19]. Disponível em: http://www.planalto.gov.br/ccivil_03/_ato2019-2022/2019/lei/L13958.htm

36. Ministério da Saúde (BR). Portaria ํo 3.510 de 18 de dezembro de 2019. Institui incentivo financeiro de custeio adicional mensal para municípios com equipe de saúde integradas à programas de formação profissional no âmbito da Atenção Primária à Saúde. Diário Oficial da União, Brasília (DF), 2019 dez. 9; Edição 245: Seção 1: 151.

37. Ministério da Saúde (BR). Portaria n 2.984, de 11 de novembro de 2019. Institui o Projeto Piloto de Apoio à Implementação da Informatização na Atenção Primária à Saúde. Diário Oficial da União, Brasília (DF), 2019 nov 13: Edição 220: Seção 1: 100. Disponível em: http://www.in.gov.br/en/web/dou/-/portaria-n-2.984-de-11-de-novembro-de-2019-227652073

38. Ministério da Saúde (BR). Portaria no 2.983, de 11 de novembro de 2019. Institui o Programa de Apoio à Informatização e Qualificação dos Dados da Atenção Primária à Saúde - Informatiza APS, por meio da alteração das Portarias de Consolidação no 5/GM/MS e nº 6/ GM/MS, de 28 de setembro de 2017. Diário Oficial da União, Brasília (DF), 2019 nov 13; Edição 220: Seção 1: 99 . Disponível em: http:// www.in.gov.br/en/web/dou/-/portaria-n-2.983-de-11-de-novembro-de-2019-227652196

39. Ministério da Saúde (BR). Ministério terá retrato da saúde da população. Brasília (DF): Ministério da Saúde; 2019 ; [acesso em 2020 jan 17]. Disponível em: http://saude.gov.br/noticias/agencia-saude/46130-ministerio-tera-retrato-da-saude-da-populacao-2

40. Ministério da Saúde (BR). Secretaria de Atenção Primária à Saúde. Fortalecimento da Atenção Primária marca os 100 dias de Saúde. Brasília (DF): Ministério da Saúde; 2019; [acesso em 2020 fev 03]. Disponível em: http://www.saude.gov.br/noticias/agencia-saude/45351-fortalecimento-da-atencao-primaria-marca-os-100-dias-de-saude-2 